\title{
Impacto de los materiales del programa de inglés en una universidad pública de Colombia*
}

\author{
MARÍA FERNANDA JAIME OSORIO** \\ CLAUDIA CAMILA CORONADO RODRÍGUEZ***
}

Recepción: 26 de marzo de 2017

Aprobación: 15 de marzo de 2018

Forma de citar este artículo: Jaime M. F. \& Coronado C. C. (2018). Impacto de los materiales del programa de inglés en una universidad pública de Colombia. Cuadernos de Lingüística Hispánica, (32), 175- 193.

\footnotetext{
* Artículo de investigación científica, derivado de un proyecto realizado por el semillero de investigación IPLEX adscrito al grupo de investigación ILESEARCH, registrado en Colciencias, Colombia.

** Magíster en Enseñanza del Inglés como Lengua Extranjera. Docente titular de la Licenciatura en Inglés, Universidad Surcolombiana, Colombia. Correo electrónico: mariafernanda.jaime@usco.edu.co.

*** Licenciada en Educación Básica con Énfasis en Humanidades, Lengua Extranjera-Inglés, Universidad Surcolombiana, Colombia Correo electrónico: clacacorodriguez@gmail.com.
} 


\section{Resumen}

Este documento es el reporte de una propuesta investigativa cuyo propósito fue diagnosticar el impacto de los materiales utilizados para la enseñanza del inglés en un curso del programa de inglés institucional de una universidad colombiana. Se acudió a los parámetros de la investigación descriptiva para analizar los materiales que se estaban implementando y a una investigación preexperimental, para determinar el impacto de nuevos y diversos tipos de materiales en el desarrollo de la habilidad oral de los estudiantes en dos grupos de cuarto nivel de inglés. Se recolectó información a través de observación de clases, diarios de campo, encuestas y entrevistas semiestructuradas a estudiantes y docentes, respectivamente. Se encontró que prevalece el uso del libro guía y materiales no-auténticos en las clases, y que a través de los nuevos materiales diseñados se benefició de manera importante la competencia oral de los estudiantes.

Palabras clave: competencia comunicativa, materiales para la enseñanza del inglés, habilidad oral.

\section{Impact of the English Program Materials in a Public University in Colombia}

\section{Abstract}

This is the report of a research proposal that had as its main purpose the identification of the impact of the materials used for teaching an English language course in the institutional English program of a Colombian university. To this end, we made use of the descriptive research parameters to analyze the materials that were being implemented in the program, and of a pre-experimental research to determine the impact of various and new types of materials in the development of oral skills into two groups of students of the fourth level of English. The information was collected through class observation, field notes, surveys, and semi-structured interviews for both students and teachers. Findings show that the course book and non-authentic materials are prevalent in classes, and that through the new materials designed by the research group, students' communicative competence was greatly developed.

Keywords: communicative competence, English teaching materials, oral skill. 


\section{Impact des matériaux du programme d'anglais dans une université publique en Colombie}

\section{Résumé}

Ce document est un rapport d'une proposition de recherche dont le propos a été celui de faire un diagnostic sur l'impact des matériaux utilisés pour l'enseignement de l'anglais, dans un cours d'anglais général d'une université colombienne. On a fait appel aux paramètres de la recherche descriptive pour analyser les matériaux qu'on utilisait, ainsi qu'à une recherche pré-expérimentale, pour déterminer l'impact des nouveaux et divers types de matériaux, dans le développement de l'habilité orale des étudiants, dans deux groupes de quatrième niveau d'anglais. On a collecté de l'information à travers l'observation des cours, des journaux de terrain, des enquêtes et des interviews semistructurées aux étudiants et aux enseignants. On a trouvé qu'il prévaut l'utilisation du manuel guide et des matériaux non-authentiques dans les cours. En plus, la compétence orale des étudiants a été bénéficiée, à travers la conception de nouveaux matériaux.

Mots clés: compétence communicative, matériaux pour l'enseignement de l'anglais, habilité orale.

\section{Impacto dos materiais do programa inglês em uma universidade pública da colômbia}

\section{Resumo}

Este documento é o relatório de uma proposta de pesquisa cujo objetivo foi diagnosticar o impacto dos materiais utilizados para 0 ensino de inglês em um curso do programa institucional de inglês de uma universidade colombiana. Os parâmetros da pesquisa descritiva foram utilizados para analisar os materiais que estavam sendo implementados e uma pesquisa pré-experimental, para determinar o impacto de novos e diversos tipos de materiais sobre 0 desenvolvimento da habilidade oral dos estudantes em dois grupos de quarto nível de Inglês. As informações foram coletadas por meio de observação de classe, diários de campo, pesquisas e entrevistas semiestruturadas com alunos e professores, respectivamente. Verificou-se que o uso do livro guia e de materiais não autênticos prevalece nas aulas, e que através dos novos materiais projetados a competência oral dos alunos beneficiou-se de maneira importante.

Palavras-chave: competência comunicativa, materiais para o ensino de inglês, habilidade oral. 


\section{Introducción}

La educación actual demanda que los profesionales universitarios adquieran competencia en la comunicación en una lengua extranjera, siendo el inglés la más popular de ellas. Sin desconocer esta demanda, el Ministerio de Educación Nacional, obedeciendo a sus propias directrices, y la Universidad Surcolombiana han establecido diferentes acuerdos. Los cursos de Interlingua están contenidos en el Acuerdo 065 del 18 de diciembre de 2009 , con el propósito de formar profesionales competitivos nacional e internacionalmente, que demuestren una competencia comunicativa en inglés, que a su vez coadyuve a la formación científica, investigativa, humanística, cultural y social de todos los estudiantes.

El desarrollo de la competencia comunicativa presupone desarrollar estas cuatro macrohabilidades: habla, escucha, escritura y lectura en la lengua extranjera, para lo cual se deben utilizar materiales apropiados que permitan al estudiante desarrollar un aprendizaje significativo a través de actividades prácticas en las que el estudiante se considere un actor central de su proceso de aprendizaje. Sin embargo, desde la creación de los cursos de inglés institucional hasta la fecha se ha utilizado un libro guía (material no auténtico, es decir, material diseñado para enseñar la lengua propiamente).

En un estudio previo realizado por Zúñiga, Insuasty, Macías, Zambrano y Guzmán (2009) se encontró que los materiales utilizados en las clases de inglés institucional fueron en un $76,1 \%$ no auténticos, es decir, materiales que se diseñaron para enseñar una segunda lengua y que

[...] el 13.6\% de los materiales tuvieron un uso mínimamente controlado, en donde el profesor proponía la actividad pero el estudiante tenía la libertad para responder, interactuar o crear, mientras un 57,6\% fueron altamente controlados reflejando así nuevamente una tendencia de carácter pre-comunicativo (sic). (p. 15)

Más adelante, Guzmán y Salazar (2009) exploraron el uso de tareas auténticas en el programa de Interlingua. Se concluyó que las tareas auténticas motivan a los estudiantes y estimulan su creatividad permitiendo así un uso real de la lengua que puede o no generar una autoevaluación del progreso en su competencia comunicativa.

A pesar de los estudios encontrados, el programa de inglés institucional, también conocido con el nombre de Interlingua, carecía de intentos recientes de intervención directa en las clases que permitieran precisamente el desarrollo de actividades menos controladas por el docente, es decir, aquellas de carácter precomunicativo que se encontró en estudios previos (Zúñiga et al., 2009; Jaime \& Insuasty, 2015). El grupo de investigación propuso entonces diseñar y evaluar el impacto de una cartilla compuesta por actividades 
que propendieran al desarrollo de la competencia oral. Para diseñar la cartilla se tuvieron en cuenta las recomendaciones dadas por Ramos y Aguirre $(2014,2016)$, quienes manifiestan la importancia de que sean los mismos docentes y los estudiantes los que diseñen sus propios materiales. Para los autores, las principales razones por las que los docentes deben diseñar materiales son la necesidad de involucrar a los estudiantes en el aprendizaje de la lengua mediante innovaciones sistemáticas, ya que así los profesores tienen en cuenta las necesidades de sus estudiantes y el desarrollo de sus habilidades, porque son los docentes los que construyen y moldean a los estudiantes a través de sus interacciones (Fajardo, 2013).

Teniendo en cuenta lo anterior, se buscó la participación activa de estudiantes de un semillero de investigación en el diseño de la cartilla, y la colaboración de los docentes que orientaban los dos cursos de inglés de cuarto nivel, a fin de explorar las debilidades y fortalezas de los materiales utilizados en el programa de Interlingua. Se propuso, entonces, un compendio de materiales suplementarios o complementarios para el cuarto nivel de inglés, con la participación de este semillero de investigación, dos docentes del programa Interlingua e investigadores del grupo ILESEARCH.

\section{Fundamentación teórica}

La referencia teórica relacionada con este proyecto está sustentada en el desarrollo de la habilidad oral, los materiales de enseñanza y la forma de diseñarlos, y los niveles de competencia del Marco Común Europeo (Consejo de Europa, 2002).

\subsection{La habilidad oral}

La habilidad oral se ha caracterizado por estar ligada a aspectos como la identidad, los estados emocionales y los factores afectivos (Hughes, 2010), lo cual la hace diferir de la habilidad de escritura, ya que quien la produce tiene más tiempo para pensar, editar y mejorar el producto escrito. A través de la habilidad oral, el individuo proyecta sus pensamientos e ideas y desarrolla su identidad como individuo y como ser social. A su vez, con la interacción, el hablante adquiere competencias para negociar significados y crear nuevas maneras de ver el mundo. Ortega y Vargas (2017) concluyen que

Las comunidades de habla se organizan alrededor de sistemas locales de conocimiento y conducta social. Tal es el caso de los adolescentes quienes están en una búsqueda continua de una identidad que los diferencie de los adultos y de los niños y se sirven de formas de comunicación particulares que se alejan, en alguna medida, de la norma estándar de la lengua. (pp. 90-91). 
El Marco Común Europeo (MCER), a su vez, clasifica la habilidad oral en dos momentos: la interacción y la producción. De acuerdo con la escala de niveles que establece este documento, en un nivel A2 se espera que el estudiante sea capaz de:

Comprender frases y expresiones de uso frecuente relacionadas con áreas de experiencia que le son especialmente relevantes (información básica sobre sí mismo y su familia, compras, lugares de interés, ocupaciones, etc.). Sabe comunicarse a la hora de llevar a cabo tareas simples y cotidianas que no requieran más que intercambios sencillos y directos de información sobre cuestiones que le son conocidas o habituales. Sabe describir en términos sencillos aspectos de su pasado y su entorno, así como cuestiones relacionadas con sus necesidades inmediatas. (Consejo de Europa, p. 26).

Asimismo, el MCER establece cinco categorías y su correspondiente descriptor, para especificar las competencias del hablante durante la producción e interacción oral:

Alcance: Utiliza estructuras compuestas por oraciones básicas con expresiones, grupos de unas pocas palabras y fórmulas memorizadas, con el fin de comunicar una información limitada en situaciones sencillas y cotidianas.

Corrección: Utiliza algunas estructuras sencillas correctamente, pero todavía comete, sistemáticamente, errores básicos.

Fluidez: Se hace entender con expresiones muy breves, aunque resultan muy evidentes las pausas, las dudas iniciales y la reformulación.

Interacción: Sabe contestar preguntas y responder a afirmaciones sencillas. Sabe indicar cuándo comprende una conversación, pero apenas comprende lo suficiente como para mantener una conversación por decisión propia.

Coherencia: Es capaz de enlazar grupos de palabras con conectores sencillos tales como «y», «pero» y «porque». (Consejo de Europa, pp. 32-33)

Para lograr el desarrollo de las competencias planteadas en el microdiseño curricular de la asignatura, los profesores del programa de inglés institucional disponen de diferentes materiales y recursos: el libro guía, guías de trabajo, grabadora, computadores, proyector, entre otros. A continuación se exponen los tipos de materiales para el desarrollo de la competencia oral. 


\subsection{Materiales para el desarrollo de la competencia oral}

Dada la popularidad del inglés como lengua materna, como segunda lengua y como lengua extranjera alrededor del mundo, hoy en día el comercio y el internet promocionan múltiples recursos y materiales que aseguran el desarrollo de la competencia comunicativa a quienes hagan uso de ellos. Hay diversas editoriales que ofrecen libros, CDS, revistas, software, videos, entre otros, para que sus usuarios aprendan nuevas palabras, practiquen el uso de ese nuevo vocabulario y mejoren su pronunciación en inglés. Sin embargo, de acuerdo con diversos autores, los materiales para la enseñanza del inglés se clasifican en dos grandes grupos: materiales auténticos y materiales no-auténticos. De acuerdo con Bacon y Finnemann (1990), los materiales auténticos son aquellos que han sido creados por hablantes nativos con propósitos no-pedagógicos.

Los beneficios del uso de materiales auténticos han sido explorados a través de diferentes estudios que han encontrado que no solo ayudan al desarrollo de la competencia comunicativa y las habilidades comunicativas de habla, escucha, lectura y escritura, sino que también tienen un impacto positivo en la motivación de los estudiantes (Gilmore, 2004; Weyers, 1999, Otte, 2006; Castillo, Insuasty \& Jaime, 2017), ya que perciben los procesos de aprendizaje de la lengua como auténticos y útiles en la vida real (McNeil, 1994; Kilickaya, 2004; Castillo et al., 2017). De esta manera, con los materiales se busca que "los profesores estimulen la reflexión, la identificación de problemas de índole social y la forma cómo solucionarlos" (Funquen \& Jiménez, 2013, p. 144).

Como se mencionó, la competencia oral es aquella que promueve el uso significativo del lenguaje, aquella que explota y desarrolla la capacidad de interacción y expresión del estudiante; por lo tanto, algunos de los materiales y actividades más relevantes para el desarrollo de la misma son las canciones, los juegos de roles, dibujos, videos, entre otros. Cada actividad debe tener un propósito específico, debe realizarse a través del uso de un material lingüístico específico, debe ejecutarse en un contexto determinado y debe involucrar al aprendiz y al profesor (Vanegas \& Zambrano, 2007). Hay que señalar que "es importante tener en cuenta los intereses de los estudiantes al planear un curso, puesto que estos llegan a ser el motor para motivar y mejorar el proceso de enseñanza-aprendizaje" (Jiménez, 2014, p. 202).

\subsection{Diseño de materiales}

A la hora de usar los materiales se ha de tener en cuenta que una lengua no es solamente un sistema de formas, estructuras y palabras, sino que desde el punto de vista pragmático es también un sistema de actos comunicativos y se espera que los medios y 
recursos empleados favorezcan el desarrollo de la competencia comunicativa del alumno, entendida como la integración de cinco subcompetencias: lingüística, sociolingüística, discursiva, cultural y estratégica (Canale, 1983). Asimismo, al diseñar materiales se recomienda considerar las pautas mencionadas por Cabalceta (2007), las cuales se basan en la psicología del color, los elementos, las siete reglas de la diagramación y las características tipográficas.

En primer lugar, según la psicología del color mencionada en el texto, se sugiere utilizar la familia del color azul para textos académicos, puesto que brinda una sensación de elegancia, sutilidad, amplitud, inteligencia y objetividad, cuyo uso puede brindarle motivación al lector. En segundo lugar, se brindan siete reglas de diagramación, las cuales están relacionadas con el uso de márgenes, tablas y columnas. También muestran el uso correcto de imágenes, espacios y texto. Todo con el propósito de brindarle al lector momentos de descanso sin distraerlo del tema central. Finalmente, se mencionan las características tipográficas, que indican que la fuente tipográfica por excelencia para textos académicos es Times New Roman, sin embargo, su uso no garantiza que el texto sea llamativo para el lector, por lo que se sugieren fuentes de tipo gótico, que son más atractivas, con gran legibilidad y versatilidad para uso académico.

Además, para el diseño de materiales se deben tener en cuenta las características propuestas por Tomlinson (1998), quien sugiere que los materiales incluyan actividades para analizar y resolver problemas, contengan temas de interés para el estudiante, varíen de acuerdo con los diferentes estilos de aprendizaje, expongan al estudiante a la lengua auténtica, impacten e inspiren confianza.

1.4 Evaluación de la competencia comunicativa y evaluación de los materiales

De acuerdo con el Marco Común Europeo, se presenta una amplia variedad de recursos evaluativos para medir la adquisición de la competencia comunicativa en una segunda lengua. En esta investigación se requirió el uso de rúbricas para evaluar la habilidad oral. Estas son consideradas parte de la evaluación formativa, la cual es descrita como un proceso en marcha que recolecta información del alcance del aprendizaje que se ha llevado a cabo en los aprendices, sus fortalezas y debilidades, en las cuales el profesor se enfoca tanto para el diseño del curso como para las correcciones que le hace a los propios estudiantes. La evaluación formativa es frecuentemente usada en muchos ámbitos, incluyendo información desconocida de cuestionarios y consultas. Su fortaleza radica en que mejora el aprendizaje en sí mismo, pero depende de la forma en que el profesor corrige y en cómo se recibe esa corrección por parte de los estudiantes. 
De alguna manera, evaluar la competencia oral de los estudiantes permite verificar la eficacia de la metodología propuesta por los materiales diseñados y, a la vez, su impacto en la motivación y el proceso formativo de los estudiantes. Por lo anterior, a efectos de evaluar los materiales, se tuvieron en cuenta los sustentos teóricos propuestos por Squires y McDougall (1997), quienes manifiestan que una perspectiva lineal y exclusivamente centrada en aspectos técnicos o puramente estéticos es baldía para la selección de los materiales que se van a aplicar en contextos educativos, siendo, en consecuencia, más partidarios de una perspectiva de evaluación colaborativa entre las diferentes personas que intervienen en el proceso de diseño, producción y utilización de los mismos; es decir, diseñadores, profesores y alumnos.

McDonough y Shaw (2003), por su parte, tienen en cuenta que se puede hacer una evaluación en general de la idoneidad de los materiales considerando los siguientes factores:

Usabilidad: que tanto el material puede ser integrado en el microdiseño curricular como "centro" o suplementario.

Generalizador: El material es adecuado para el trabajo individual y en grupo.

Adaptabilidad: El material puede ser adicional, extraído o usado en otro contexto 0 modificado para otras circunstancias.

Flexibilidad: Algunos materiales ofrecen una medida de flexibilidad que les permite integrarse fácilmente en varios tipos de programas. (p. 73)

Teniendo en cuenta lo anterior, este estudio investigativo empleó la rúbrica en la aplicación de un pretest y un postest para cuantificar la habilidad oral de los estudiantes, y aplicó instrumentos como las encuestas para conocer la percepción de los docentes y los estudiantes en cuanto a los beneficios identificados de los materiales propuestos por el grupo de investigación.

\section{Metodología de investigación}

El proyecto se desarrolló dentro de los parámetros del modelo de investigación mixta, ya que se llevaron a cabo tareas descriptivas, cualitativas y de índole preexperimental, las cuales se usaron en diferentes etapas del proceso.

En la primera etapa se seleccionaron dos grupos de cuarto nivel de Interlingua, el grupo control con 35 estudiantes y el grupo experimental con 38 estudiantes, que se encuentran en un rango de edad entre los 18 y 24 años. Luego se hicieron cuatro obser- 
vaciones de clases y se aplicó el pretest a una muestra de diez estudiantes de cada grupo, con el que se evaluó la habilidad oral de estos. También se encuestaron los seis docentes del cuarto nivel para identificar los materiales más utilizados por ellos en el desarrollo de sus clases. En la segunda etapa, a partir de las observaciones de clases y el pretest, se diseñó y aplicó un compendio de materiales suplementarios, cuyo objetivo principal era desarrollar la habilidad oral de los estudiantes. En la tercera etapa se realizaron seis observaciones de clases, en las que se aplicaron los materiales complementarios y se implementó el postest. Finalmente, en la cuarta etapa se analizaron los datos recopilados durante la investigación.

\section{Resultados, análisis y discusión}

El propósito de esta investigación era identificar el impacto del uso de materiales utilizados para el desarrollo de la habilidad oral de los estudiantes de un curso de cuarto nivel del programa de inglés institucional de una universidad colombiana. Para ello, identificamos y clasificamos los materiales que se estaban empleando para la enseñanza de esta lengua, se diseñó un compendio de materiales complementarios y se evaluó su impacto en el desarrollo de la competencia oral de los estudiantes. A continuación, se presentarán los principales hallazgos obtenidos durante la investigación.

\subsection{Pretest y postest}

Para explorar el nivel de competencia oral de los estudiantes participantes se aplicó un pretest a una muestra de diez estudiantes en cada grupo, el experimental y el de control. El puntaje máximo obtenido por los estudiantes en el dominio de cada uno de estos criterios fue 3 (100\%). La Tabla 1 muestra un cuadro comparativo de los resultados del pretest y del postest en el grupo experimental y el de control. Con resultados similares en alcance y coherencia, los grupos evidenciaron diferencias significativas en corrección, fluidez e interacción en los resultados del pretest. En general, el grupo de control presentaba un desarrollo más elevado de cada uno de los criterios de la competencia oral al iniciar el semestre académico (periodo B-2016). 
Tabla 1. Resultados pretest y postest grupo experimental y grupo de control

\begin{tabular}{|l|c|c|c|c|c|c|c|c|}
\hline \multirow{2}{*}{ Criterio } & \multicolumn{4}{|c|}{ Grupo Experimental } & \multicolumn{4}{c|}{ Grupo de control } \\
\cline { 2 - 9 } & $\begin{array}{c}\text { Promedio } \\
\text { Pretest }\end{array}$ & $\%$ & $\begin{array}{c}\text { Promedio } \\
\text { Postest }\end{array}$ & $\%$ & $\begin{array}{c}\text { Promedio } \\
\text { Pretest }\end{array}$ & $\%$ & $\begin{array}{c}\text { Promedio } \\
\text { Postest }\end{array}$ & $\%$ \\
\hline Alcance & 1,8 & $60 \%$ & 1,7 & $55 \%$ & 1,8 & $60 \%$ & 1,8 & $60 \%$ \\
\hline Corrección & 1,4 & $47 \%$ & 2 & $66 \%$ & 2,1 & $70 \%$ & 2,2 & $73 \%$ \\
\hline Fluidez & 1,8 & $60 \%$ & 2,1 & $70 \%$ & 2,1 & $70 \%$ & 1,9 & $63 \%$ \\
\hline Interacción & 1,7 & $55 \%$ & 2,2 & $73 \%$ & 2,3 & $77 \%$ & 1,9 & $63 \%$ \\
\hline Coherencia & 1,5 & $50 \%$ & 1,9 & $63 \%$ & 1,7 & $57 \%$ & 1,9 & $63 \%$ \\
\hline
\end{tabular}

En comparación con los resultados obtenidos en el pretest, los resultados del postest muestran que esta vez el grupo experimental presentó una leve elevación en su competencia oral en todos los criterios, exceptuando el de alcance, que disminuyó una décima. El criterio en el que demuestra mayor dominio es el de interacción, pero es el criterio de corrección el que presenta mayor elevación en el promedio. Esto denota una mayor conciencia de los estudiantes en la producción oral con errores y la capacidad que tienen para autocorregirse en el mismo momento en que producen el error.

Por otra parte, el grupo de control, el cual no tuvo intervención alguna por parte de este grupo de investigación, mejoró en el promedio en los criterios de corrección y coherencia, mientras que el promedio de dominio en el alcance se mantuvo y desmejoró en fluidez e interacción. Teniendo en cuenta que los estudiantes del grupo de control habían iniciado con resultados ventajosos en el pretest en comparación con sus pares del grupo experimental, se puede concluir que la intervención directa a través de la estrategia de uso de los materiales diseñados por los investigadores con participación de estudiantes y docentes causó un efecto positivo en el desarrollo de la competencia oral.

\subsection{Prevalencia del libro guía en las clases y sus implicaciones}

Para identificar el tipo de materiales que se estaban empleando en las clases de inglés, se recolectó información desde varias fuentes, siendo dos de estas la encuesta A para docentes y la encuesta A para estudiantes.

La Figura 1 representa las respuestas a la pregunta número 1 de la encuesta a docentes, quienes manifiestan que los materiales más utilizados por ellos en el desarrollo de sus clases de inglés son la cartilla y sus respectivas grabaciones (17\%). Seguidamente, un $15 \%$ de los encuestados manifestó hacer uso de fotos y otros materiales entre los que mencionaron la plataforma educativa Edmodo, películas, láminas, juegos y fotocopias con 
ejercicios. Otros materiales utilizados con menor frecuencia en el aula son las canciones, los pósteres y las conferencias.

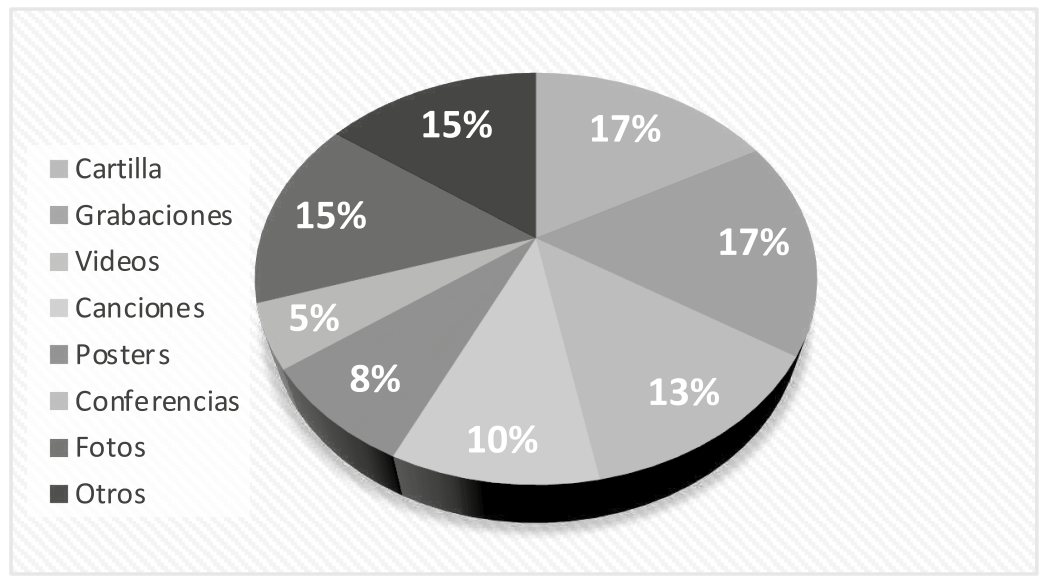

Figura 1. Materiales empleados en la clase de inglés.

El $100 \%$ de los estudiantes y docentes encuestados respondió que el libro guía es el material sobre el cual basan la enseñanza de la lengua para este nivel específico. Asimismo, esto se evidenció en las observaciones de clases, donde el libro guía aparece como el material central para la enseñanza-aprendizaje del inglés. Varios autores han mencionado los beneficios del libro guía (Aciega, 2007), pues este es uno de los recursos que permiten al docente establecer una relación personal, en la que se revaloriza el diálogo para una mejor interacción entre los actores del proceso de enseñanza-aprendizaje y el desarrollo de la habilidad oral. Sin embargo, la información recolectada en esta investigación permitió conocer la percepción que tienen docentes y estudiantes con respecto al libro guía utilizado.

La Figura 2 muestra las respuestas de los docentes y los estudiantes a dos de las preguntas de las encuestas. La pregunta dos (P2) indaga sobre la utilidad de los materiales utilizados por los docentes para el desarrollo de sus clases. Es de resaltar que a pesar de gozar de buen concepto ante los estudiantes ( $83 \%$ contestaron que los materiales, entre los que distinguimos videos, fotos, sí son apropiados), el $43 \%$ de los docentes y el $17 \%$ de los estudiantes encuentran el libro guía poco apropiado para desarrollar la competencia comunicativa y destacan que no perciben desarrollo de la competencia oral a través de los ejercicios propuestos por este. A diferencia de la pregunta dos (P2), cuando se les pregunta a los docentes y a los estudiantes sobre la pertinencia de la cartilla de inglés (material en el que basan la enseñanza los docentes), ambos tienen percepciones encontradas. Mientras que el $100 \%$ de los docentes encuestados no la encuentra pertinente, el $92 \%$ de los estudiantes sí. 


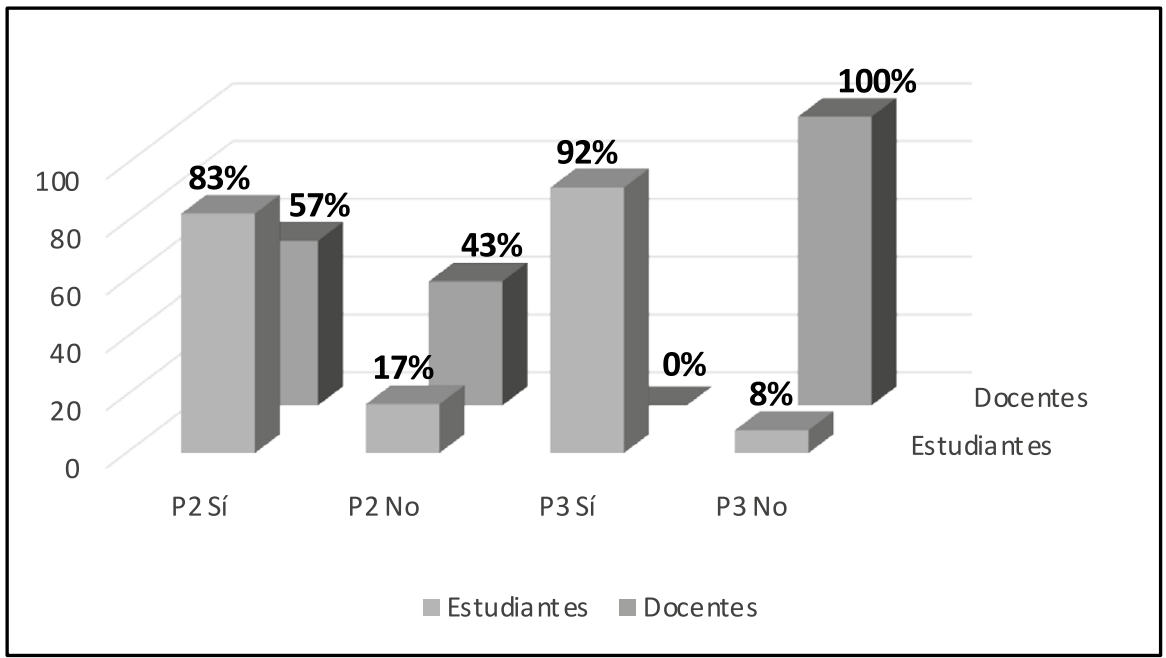

Nota: los valores expresados en porcentajes representan el total de población encuestada: Docentes $=8 \mathrm{y}$ Estudiantes $=65$.

Figura 2. Percepción estudiantes-docentes utilidad de los materiales de inglés y su pertinencia para el desarrollo de la competencia comunicativa.

Las razones enunciadas por los docentes encuestados varían desde la desactualización del libro y el microdiseño curricular (los cuales fueron propuestos en el año 2005, es decir, hace once años), el enfoque gramatical del libro, la descontextualización de las actividades, la insuficiencia de ejercicios orales y la incoherencia de la estructura del libro, dado que no presenta un lineamiento claro sobre cómo desarrollar la habilidad oral en particular.

Por otra parte, los estudiantes encuestados, quienes en su mayoría perciben el libro guía como pertinente para el aprendizaje de la lengua inglesa, indicaron entre otras las siguientes razones:

- S1. "Porque con ella (la cartilla) podemos practicar inglés oral, escucha y escritura. Contiene todos (tipos de ejercicios para desarrollar la competencia comunicativa), son claros, concisos y entendibles".

- S2. "Porque se interacciona con toda clase de herramientas que desarrollan todas las competencias del saber- oír- hablar- escribir- leer".

- S3. "Porque podemos interactuar de una forma más cotidiana en la cual se puede hacer 0 aplicar fuera de la clase, ejemplo: videos pero aún faltan mejores materiales". 
Aunque la mayoría de los comentarios de los estudiantes encuestados hace alusión al desarrollo de la competencia comunicativa y la calidad de los audios, existe un porcentaje importante de estudiantes que percibe que los materiales no son los adecuados (17\%), que no desarrollan su competencia oral (19\%) ni su competencia comunicativa (8\%). Algunas de las razones enunciadas se basan en la falta de elementos didácticos en los ejercicios propuestos en los materiales, de apoyo audiovisual y métodos que faciliten la integración e interiorización de la información, así como en la falta de ejercicios que reflejen las diferencias culturales del lenguaje; igualmente señalaron la desactualización del libro.

Los resultados mencionados nos llevaron a hacer un análisis del texto guía teniendo como herramienta una lista de chequeo adaptada de la propuesta por Peacock (1997), en la que se evaluaron ocho aspectos: la impresión general, la calidad técnica, las diferencias culturales, la adecuación, la motivación y el alumno, el análisis pedagógico, encontrar el camino a través del libro del estudiante y los materiales suplementarios. Se encontró que el libro tiene diversos aspectos positivos, entre los que se destacan el desarrollo de las cuatro habilidades lingüísticas, la calidad de los audios y la disponibilidad o acceso al libro. Asimismo se confirmaron algunos aspectos poco favorables ya manifestados por docentes y estudiantes, como la baja motivación que genera, la calidad técnica de las fotocopias que adquieren los estudiantes (cuando deciden no comprar el libro original), y la poca exposición a diferencias culturales.

Teniendo en cuenta los resultados anteriores, es necesario, a petición de los mismos docentes y estudiantes, que se complemente la utilización del libro guía con actividades y materiales que propendan al desarrollo de la competencia comunicativa y cultural de los estudiantes. Se debe resaltar, por lo tanto, el esfuerzo que hacen aquellos docentes, pues, a pesar de factores adversos como el tiempo de las clases (cuatro horas semanales) y el número de los estudiantes (que varía entre 30 y 45 por curso), promueven un aprendizaje más significativo a través del uso de las tecnologías de la información y la comunicación (TIC) y materiales complementarios diseñados por ellos mismos.

\subsection{El diseño, aplicación e impacto de los materiales complementarios}

A partir de los resultados de las observaciones y las encuestas realizadas, el equipo de estudiantes investigadores diseñó un compendio de materiales complementarios que desarrollaron la competencia oral de los estudiantes de cuarto nivel de Interlingua. Para ello, se tuvo en cuenta que los materiales debían ser recursos que facilitaran el proceso de aprendizaje y enseñanza, en el que se debía promover la adquisición de habilidades y destrezas (Ogalde \& Bardavid, 2003). 
Para la realización del diseño de los materiales, se consideraron los resultados de encuesta A para estudiantes, la cual determinó que los juegos y los debates son las actividades de mayor preferencia por los estudiantes para desarrollar la habilidad oral. A partir de los intereses de los estudiantes, las necesidades del curso y la experiencia del docente, se creó un libro de actividades que contenía 14 actividades (juegos, canciones, debates, historias, entre otros). Para ello, se revisó minuciosamente el microdiseño curricular del curso para que las actividades fueran apropiadas y tuvieran relación directa con el libro. Debido a esto, se adecuaron algunas canciones, juegos y debates con las estructuras gramaticales enseñadas en clase, partiendo del contexto educativo regional y nacional. Por ejemplo, para las discusiones se diseñó una hoja guía en que se describe el tipo de actividad, el objetivo de esta, el tiempo y las instrucciones para el desarrollo de la actividad. El tema del medio ambiente incluía la presentación de un video como referencia para generar un debate entre los participantes a través de preguntas como What do you think about global warming? Do you think it is real?

What can you do to avoid the damage against nature? Have you suffered any global warming consequences recently? If so, how?

Los materiales complementarios que se diseñaron abordaron temáticas actuales que le permitían a los estudiantes contextualizarlas. Al evaluarse el impacto de los nuevos materiales se observaron los resultados del postest (ver 3.1), de las observaciones de clase y de las encuestas B a docentes y estudiantes. A través de la triangulación de la información obtenida por medio de los instrumentos aplicados, se pudo determinar que el uso de los materiales diseñados impactó positivamente el desarrollo de la competencia oral y la motivación de los estudiantes. Para dicho examen se consideraron las cinco categorías propuestas por el Marco Común Europeo, en las que especifican las competencias del hablante durante la producción e interacción oral. Las competencias evaluadas fueron alcance, corrección, fluidez, interacción y coherencia.

En la aplicación del postest, por ejemplo, se evidenció que, en efecto, el desarrollo de la competencia oral de los estudiantes del grupo experimental se elevó y sobrepasó la de los estudiantes del grupo de control. Estos resultados fueron corroborados por las observaciones de clase hechas durante las seis aplicaciones y los resultados de las encuestas a los estudiantes y el docente del grupo experimental. Si bien los estudiantes aún presentan inconvenientes a la hora de poner en práctica sus conocimientos, ahora son más conscientes de los errores y hacen lo posible por utilizar el inglés al realizar las actividades propuestas en los materiales diseñados. Esto garantiza que los estudiantes respondan de manera au- 
téntica a las tareas ejercidas durante la clase y que su dominio de la competencia oral se vea incrementado.

A pesar de que el $19 \%$ de los estudiantes cree que los materiales diseñados por el equipo de investigación e implementados por el docente no son los más apropiados para el desarrollo de la competencia oral, de acuerdo con los resultados obtenidos es importante resaltar que el 88 \% de los estudiantes calificó los materiales a partir de tres categorías: excelentes, muy buenos o buenos. También manifiestan que tienen un impacto positivo en el desarrollo de su competencia oral, siendo el debate la actividad que consideran más beneficiosa. Parece contradictorio, pero se entiende que la percepción de que el material sea apropiado o no, se desliga del concepto de la calidad de este; es decir, el material puede ser excelente o muy bueno, pero no significa que sea considerado el más apropiado por los estudiantes.

Otros factores que emergieron durante la aplicación de los materiales y la evaluación de su impacto fueron, en primer lugar, la inasistencia de algunos estudiantes, lo que afectó el desarrollo de la competencia oral de aquellos estudiantes que no asistían con regularidad al curso. Y en segundo lugar, el docente del grupo experimental no incentivaba a los estudiantes a emplear el inglés como lengua extranjera durante la aplicación de los materiales complementarios, ya que después de hacer intervenciones en inglés, traducía lo que había dicho, lo que hacía que los estudiantes respondieran en español, situación que, por supuesto, también afectó el desarrollo de la competencia oral de los estudiantes de cuarto nivel de Interlingua.

\section{Conclusiones}

El objetivo principal de la investigación era diagnosticar el impacto de los materiales utilizados para el desarrollo de la habilidad oral de los estudiantes de un curso de cuarto nivel del programa de Interlingua de la Universidad Surcolombiana, a partir de la aplicación de instrumentos como encuestas. Se identificó que los materiales más empleados en la enseñanza del inglés en el programa de Interlingua son el libro guía, fotocopias para explicación gramatical y canciones.

Para Hughes (2010), la habilidad oral se ha caracterizado por estar ligada a aspectos como la identidad, los estados emocionales y factores afectivos. A través de la interacción, el hablante adquiere competencias para negociar significados y crear nuevas maneras de ver el mundo. Debido a esto y de acuerdo con los resultados de los instrumentos, se concluye que la prevalencia del libro guía utilizado no favorece un desarrollo significativo de la 
habilidad oral, debido a factores como la calidad de las actividades y la falta de aplicación de ejercicios orales que propendan a la producción y la interacción.

En cuanto al diseño y aplicación de materiales complementarios, se encontró que favorecen la práctica pedagógica del docente, especialmente cuando se involucra a este en la creación de los mismos (Ramos \& Aguirre, 2014), ya que se crearon actividades llamativas que motivaron a los estudiantes y ayudaron al mejoramiento y desarrollo de la habilidad oral. Es necesario entonces reconocer la importancia que tiene la creación de materiales complementarios a los cursos de inglés institucional de la Universidad Surcolombiana, que contengan características que correspondan a las necesidades particulares del contexto educativo en el que se enseña y a los objetivos específicos establecidos por la institución educativa para el nivel propuesto. Para dinamizar las prácticas pedagógicas de los docentes se recomienda, además de la creación de materiales complementarios, la enseñanza reflexiva del docente, para que sea él mismo quien identifique las necesidades próximas de sus estudiantes y las estrategias que mejorarán su desempeño y el logro de los objetivos propuestos para cada una de sus clases.

En último término, se espera que los resultados de esta investigación sean beneficiosos para la Universidad Surcolombiana, en la medida que se propongan cambios sustantivos en la selección del libro guía y en las orientaciones curriculares proporcionadas a los docentes de los cursos de inglés institucional. También se invita a docentes de otras universidades con programas de inglés institucional similares a este, para que realicen investigaciones en esta área y complementen de manera significativa los hallazgos.

\section{Referencias}

Aciega, D. (2007). Los libros de texto para la enseñanza de inglés: una mirada crítica, memoria académica. Puertas Abiertas 3(3), 99-102. Recuperado de http://www.memoria.fahce. unlp.edu.ar/art_revistas/pr.4582/pr.4582.pdf

Bacon, S., \& Finneman, M. (1990). A Study of Attitudes, Motives, and Strategies of University Foreign Language Students and their Disposition to Authentic Oral and Written Input. Modern Language Journal, 74(4), 459-73.

Cabalceta, I. (2007). Manual de diseño y diagramación de documentos. Ministerio de Educación Pública de Costa Rica. Unidad de Material Impreso. Recuperado de http://www. colombiaaprende.edu.co/html/mediateca/1607/articles-168315_archivo.pdf

Canale, M. (1983). From Communicative Competence to Communicative Language Pedagogy. In J. C. Richards \& R. W. Schmidt (eds), Language and communication (pp. 2-14). London: Longman. 
Castillo, C.A., Insuasty, E.A., \& Jaime, M.F. (2017). The Impact of Authentic Materials and Tasks on Students' Communicative Competence at a Colombian Language School. Profile Issues in Teachers' Professional Development, 19(1), 809-104. http://dx.doi.org/10.15446/profile. v19n1.56763.

Consejo de Europa. (2002). Marco Común Europeo de referencia para las lenguas: aprendizaje, enseñanza, evaluación. Madrid: MECD y Anaya. Recuperado de http://cvc.cervantes.es/ obref/marco

Fajardo, J.A. (2013). What Makes a Teacher: Identity and Classroom Talk. Cuadernos de Lingüística Hispánica, (22), 127-146. Retrieved from http://revistas.uptc.edu.co/index. php/linguistica_hispanica/article/view/2159/5178

Fúnquen, A. \& Jiménez, P.K. (2013). Implementing Tasks that Stimulate Critical Thinking in EFL Classrooms. Cuadernos de Lingüística Hispánica, (21), 143 - 158. https://doi. org/10.19053/0121053X.1955

Gilmore, A. (2004). A Comparison of Textbook and Authentic Interactions. ELT Journal, 58(4), 363-374.

Guzmán, N. \& Salazar, S. A. (2009). Análisis de las prácticas pedagógicas de los docentes de inglés del programa interlingua. Estudio diagnóstico. Manuscrito no publicado. Facultad de Educación, Universidad Surcolombiana, Neiva, Colombia.

Hughes, R. (2010). Materials to Develop the Speaking Skill. In N. Harwood (ed.), English Language Teaching Materials: Theory and Practice (pp. 207-224). Cambridge: University Cambridge Press.

Jaime, M. F. \& Insuasty, E. A. (2015). Analysis of the Teaching Practices at a Colombian Foreign Language Institute and their Effects on Students' Communicative Competence. How, 22(1), 45-64.

Jiménez, P. (2014). Exploring Students' Reactions when Working Teaching Materials Designed on their Own Interests. Cuadernos de Lingüística Hispánica, (25) 201-222. Retrieved from http://revistas.uptc.edu.co/revistas/index.php/inguistica_hispanica/article/ view/3378/4958

Kilickaya, F. (2004). Authentic Materials and Cultural Content in EFL Classrooms. The Internet TESL Journal, 10(7).

McDonough, J. \& Shaw, C. (2003). Materials and Methods in ELT, Teacher's Guide, (2 ${ }^{\text {nd }}$ ed.), Hoboken, NJ: Blackwell. 
McNeil, A. (1994). What Makes Authentic Materials Different? The Case of English Language Materials for Educational Television. In Papers presented at the Annual International Language in Education Conference, Hong Kong.

Ogalde, I. \& Bardavid, E. (2003). Los materiales didácticos. Medios y recursos de apoyo a la docencia. (2. ${ }^{\mathrm{a}}$ ed.). México: Trillas.

Ortega, F.J. \& Vargas, B.V. (2017). Aproximación sociopragmática a las estrategias conversacionales de los adolescentes. Cuadernos de Lingüística Hispánica, (29), 83-103. http://dx.doi. org/10.19053/0121053X.n29.2017.5849

Otte, J. (2006). Real Language to Real People: A Descriptive and Exploratory Case Study of the Outcomes of Aural Authentic Texts on the Listening Comprehension of Adult ESL Students Enrolled in an Advanced ESL Listening Course. Dissertation Abstracts International, $67(4), 1246 \mathrm{~B}$.

Peacock, M. (1997). Choosing the Right Book for Your Class. Retrieved from http://www.sx.ac.uk/ linguistics/pgr/egspll/volume1/ PDFs/PEACOCK1.pdf

Ramos, B. \& Aguirre J. (2014). Materials Development in the Colombian Context: Some Considerations about its Benefits and Challenges. How, 21(2), 134-150.

Ramos, B. \& Aguirre, J. (2016). English Language Teaching in Rural Areas: A New Challenge for English Language Teachers in Colombia. Cuadernos de Lingüística Hispánica, (27), 209222.

Squires, D. \& McDougall, A. (1997). Cómo elegir y utilizar software educativo. Madrid: Morata.

Tomlinson, B. (1998). Materials Development in Language Teaching. Cambridge: Cambridge University Press.

Vanegas, L. E. \& Zambrano, L. (2007). Cómo enseñar inglés en primaria: una propuesta metodológica. Neiva, Colombia: Corpus Litografía.

Weyers, J. (1999). The Effect of Authentic Video on Communicative Competence. The Modern Language Journal, 83(3), 339-349. http://dx.doi.org/10.1111/0026-7902.00026

Zúñiga, G., Insuasty, E. A., Macías, D. F., Zambrano, L. C. \& Guzmán, N. (2009). Análisis de las prácticas pedagógicas de los docentes de inglés del programa interlingua de la Universidad Surcolombiana. Revista Entornos, 22, 11-20. 